\title{
Steady shear behavior of polymerically stabilized suspensions: Experiments and lubrication based modeling
}

\author{
P. A. Nommensen, M. H. G. Duits, D. van den Ende, and J. Mellema \\ Rheology Group, Department of Applied Physics, University of Twente, P.O. Box 217, 7500 AE, Enschede, The Netherlands
}

(Received 26 August 1998)

\begin{abstract}
Flow curve measurements are presented of a suspension of polymerically stabilized monodisperse spheres, with a polymer layer thickness of 0.7 times the core radius. At low shear rates a drastic change in behavior occurs at a critical (effective) volume fraction $\phi_{m}$. Below $\phi_{m}$ the curves show a low shear Newtonian plateau. The concentration dependence of these plateaus together with $\phi_{m}=0.60$ indicate that the particles can be modeled as Brownian hard spheres. Above this $\phi_{m}$ the flow curves indicate plastic behavior, due to the direct contact between polymer layers of different spheres. At high shear rates the onset of Newtonian plateaus is observed with a gradual concentration dependence. The experimental high shear data are compared with model calculations, based on the dissipation due to lubrication forces treating the polymer layer as a Brinkman medium. The model we used was adapted to give a better description of the polymer layer. Fitting the experiments to obtain the permeability of the polymer layer, we found a value in the range of values expected from polymer theory. Our adapted lubrication model was also used to reanalyze experimental data for other polymerically stabilized suspensions. [S1063-651X(99)02703-8]
\end{abstract}

PACS number(s): $82.70 . \mathrm{Kj}, 36.20 .-\mathrm{r}$

\section{INTRODUCTION}

To understand the rheology of suspensions of sterically stabilized spheres, it is in many cases a justified first approach to describe the particles as (effective) hard spheres [1]. However, the properties of the stabilizing layer on the surface of the particles will eventually manifest themselves under the proper conditions like, e.g., particle concentration or shear rate. Due to the finite thickness of the stabilizing layer, deviations from hard-sphere rheology can be expected since the stabilizing layer is deformable and the solvent can penetrate into this layer.

Sterically stabilized suspensions were studied experimentally for these deviations by D'Haene [2], Jones et al. [3] and Neuhäusler and Richtering [4]. The stabilizing layers in these studies are, however, either relatively thin $[2,3]$ or consist of adsorbed polymers that allow changes in the number of polymers in the layer so the layer properties can change during measurement [4].

On the theoretical side only recently the first attempts were reported, which give a description for steady shear behavior that really focus on the properties of the stabilizing layer. Such models were reported by Fredrickson and Pincus [5] and Potanin and Russel [6]. The last model describes the dominant hydrodynamic interaction of sterically stabilized particles over a range of particle separations. It takes into account that the solvent can penetrate into the stabilizing layer but it does not describe the limiting behavior for dense brushes in the proper way.

This paper comprises a contribution to the mentioned field of interest, experimentally as well as theoretically. We present the results and modeling of steady shear rate viscosity measurements on suspensions consisting of silica cores coated with a brush of end-grafted poly(dimethylsiloxane) (PDMS) chains. This particle system was made in our own laboratory and was selected for a number of reasons. The size distribution for the silica cores can be kept narrow. The grafting is performed in a separate procedure, which allows a more elaborate characterization of the particles. The polymer chains are chemically attached to the cores, which prevents changes in layer properties. The molecular weight of the polymer chains is high, which results in a thick stabilizing layer where the individual chains still have polymeric properties. These properties make it a well-defined system to investigate the influence of the polymer layer on the rheology of the particles. In this paper we concentrate on the flow curves of this system with special emphasis on the limiting cases of low and high shear rate behavior. For the high shear rate regime we modified the model of Potanin and Russel and compared it to our experimental data and those of D'Haene and Neuhäusler and Richtering.

This paper is organized as follows: In the next section the modifications of the model of Potanin and Russel [6] will be discussed. In Sec. III the suspension characterization and the details of the rheological measurements are treated. The discussion in Sec. IV has been split up in three parts based on the range of shear rate. In the low shear rate part and the shear thinning part our results are compared to other systems and to empirical relations. In the last part, results of calculations based on the model described in Sec. II are compared to the measured high shear viscosities of our systems as well as other systems. In Sec. V conclusions will be drawn.

\section{LUBRICATION THEORY}

\section{A. Lubrication force between two spheres}

Our description of lubrication forces between hairy spheres is based on the work of Potanin and Russel [6] (thereafter referred to as PR). In this section the key equations are repeated for the convenience of the reader. We will also discuss some modifications.

In this description two particles with core radius $R_{c}$ and polymer layer thickness $L$ and core-core surface separation $H$ 


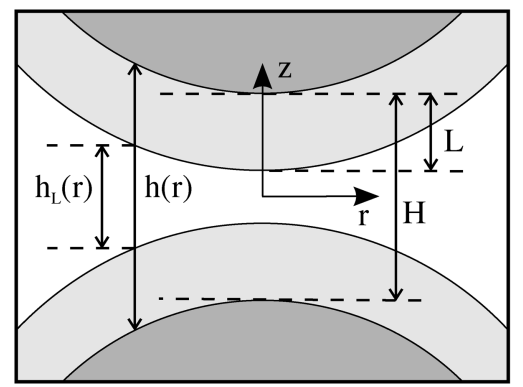

FIG. 1. Hydrodynamically interacting particles surrounded by a permeable layer.

move to each other with a velocity $V \equiv-d H / d t$, see Fig. 1 . It is convenient to describe the resulting flow problem in cylindrical coordinates with the origin in the middle of the gap between the particles. When they are close together, the hydrodynamic force on the particles is dominated by the pressure difference in and outside the gap and can be calculated by integrating the pressure field over the symmetry plane $z=0[6]$ :

$$
F=2 \pi \int_{0}^{\infty} r\left[p(r)-p_{\infty}\right] d r=-\pi \int_{0}^{\infty} r^{2} \frac{\partial p}{\partial r} d r .
$$

The pressure gradient is caused by the particles when squeezing the solvent out of the gap. By modeling the polymer layer as a Brinkman medium with permeability $\delta^{2}$, the squeezing flow field can be calculated, where the magnitude of $\delta^{2}$ reflects properties of the polymer layer like the monomer density.

The Brinkman equations can be simplified when the gap thickness $H$ is much smaller than $R_{c}$ :

$$
\begin{gathered}
\frac{1}{r} \frac{\partial}{\partial r}\left(r v_{r}\right)+\frac{\partial}{\partial z} v_{z}=0, \\
\frac{\partial}{\partial z} p=0 \\
\frac{\partial^{2}}{\partial z^{2}} v_{r}-\frac{1}{\delta^{2}} v_{r}=\frac{1}{\mu} \frac{\partial}{\partial r} p,
\end{gathered}
$$

where $\mu$ is the solvent viscosity, $v_{r}$ and $v_{z}$ are the velocity in, respectively, the radial and axial direction. To calculate the hydrodynamic force (1) first $\partial p / \partial r$ has to be calculated from Eq. (4). In the limit $H / R_{c} \ll 1$, the pressure is independent of $z$. Therefore, the velocity $v_{r}$ is made dimensionless by

$$
\bar{v}_{r}(\bar{r}, \bar{z}) \equiv \frac{v_{r}(r, z)}{-\frac{H^{2}}{\mu} \frac{\partial p}{\partial r}},
$$

while $r$ and $z$ are scaled according to

$$
\bar{r} \equiv \frac{r}{\sqrt{H R_{c}}}, \quad \bar{z} \equiv \frac{z}{H} .
$$

By applying these scaling relations a more simple form for Eq. (4) is found,

$$
\frac{\partial^{2}}{\partial \bar{z}^{2}} \bar{v}_{r}-\frac{H^{2}}{\delta^{2}} \bar{v}_{r}=-1
$$

The boundary conditions are $\bar{v}_{r}=0$ at $\bar{z}=\bar{h} / 2$ (no slip condition at the core surface) and $\partial \bar{v}_{r} / \partial \bar{z}=0$ at $\bar{z}=0$ due to symmetry. Since the permeability $\delta^{2}$ is very different in and outside the polymer layer it is straightforward to solve Eq. (7) separately in these regions and link the solutions together with the extra boundary conditions that both $\bar{v}_{r}$ and $\partial \bar{v}_{r} / \partial \bar{z}$ are continuous at the polymer-solvent interface $(\bar{z}$ $\left.=h_{L} / 2 H\right)$. The local gap thickness $h$ and the position of the interface $h_{L} / 2$ as a function of $r$ are given by

$$
\begin{aligned}
h(r) & =H+2 R_{c}-2 \sqrt{R_{c}^{2}-r^{2}}, \\
& \approx H+\frac{r^{2}}{R_{c}}+O\left(r^{4}\right), \\
h_{L}(r) & =H+2 R_{c}-2 \sqrt{\left(R_{c}+L\right)^{2}-r^{2}}, \\
& \approx H-2 L+\frac{r^{2}}{R_{c}+L}+O\left(r^{4}\right) .
\end{aligned}
$$

Here the core surface and the interface are described as two concentric spheres with radii, respectively, $R_{c}$ and $R_{c}+L$. It differs from the description PR used, in which the core surface and the interface are defined by two spheres with same radius but with the centers shifted over a distance $L$, i.e., $h_{L}=h-2 L$. In their description the size of the polymer layer is underestimated for nonzero $r$, which tends to be an underestimation of the lubrication force.

The permeability in Eq. (7) depends on the monomer density, which for polymers attached to spherical surfaces, is a function of $\bar{z}$ and $\bar{r}$. Following PR we assume a step profile for the monomer density making the permeability constant $\left(\delta=\delta_{0}\right)$ in uncompressed areas and a function of the ratio compressed and uncompressed layer thickness in compressed areas,

$$
\delta=\delta_{o}\left(\frac{h}{h-h_{L}}\right)^{n}, \quad h_{L}<0
$$

where $n$ reflects the solvent quality, $n=\frac{3}{4}$ for good solvents and $n=1$ for theta solvents.

Solving Eq. (7) with Eq. (10) as a substitution for $\delta$ and Eqs. (8) and (9) in the boundary conditions, the dimensionless velocity $\bar{v}_{r}(\bar{r}, \bar{z})$ is known. This result is used to obtain an expression for $\partial p / \partial r$ by integrating Eq. (5) over $z$ on both sides, which results in

$$
\frac{\partial p}{\partial r}=-\frac{\mu}{H^{3}} \frac{\int_{0}^{h(r) / 2} v_{r} d z}{\int_{0}^{\bar{h}(r) / 2} \bar{v}_{r} d \bar{z}}=-\frac{\mu V}{4 H^{3}} \frac{r}{\bar{Q}(\bar{r})},
$$


where conservation of mass [i.e. Eq. (2)] is used to find

$$
\int_{0}^{h(r) / 2} v_{r} d z=\frac{V r}{4}
$$

and $\bar{Q}$ is defined as

$$
\bar{Q}(\bar{r}) \equiv \int_{0}^{\bar{h}(\bar{r}) / 2} \bar{v}(\bar{r}, \bar{z}) d \bar{z} .
$$

$\bar{h}$ is the local gap thickness scaled by $H$. Substituting Eq. (11) in Eq. (1) gives a representation for the hydrodynamic force with one integration less than the one given by PR:

$$
\bar{F}=\frac{F}{6 \pi \mu R_{c} V}=\frac{R_{c}}{24 H} \int_{0}^{\infty} \frac{\bar{r}^{3}}{\bar{Q}(\bar{r})} d \bar{r} .
$$

\section{B. Suspension viscosity as a function of concentration}

The lubrication force can be used to obtain the highfrequency viscosity. Frankel and Acrivos [7] calculated the contribution of lubrication to the viscosity under the assumptions that the particle pair distribution function is characterized by the mean intercenter distance $\left\langle R_{12}\right\rangle$ between neighboring particles and that the motion of the spheres is affine. They derived the expression,

$$
\frac{\mu_{\text {lub }}}{\mu}=9 \frac{2 R_{c}}{2 R_{c}+H_{\mathrm{av}}} \bar{F}\left(H_{\mathrm{av}}\right)
$$

with $H_{\mathrm{av}}=\left\langle R_{12}\right\rangle-2 R_{c}$, the mean separation between core surfaces. It can be related to the volume fraction of the cores $\phi_{c}$ using a geometrical argument,

$$
H_{\mathrm{av}}=2 R_{c}\left[\left(\frac{\phi_{\mathrm{max}}^{\mathrm{core}}}{\phi_{\text {core }}}\right)^{1 / 3}-1\right],
$$

where $\phi_{\max }^{\text {core }}$ is the maximum volume fraction of the cores corresponding to a particle configuration with $H=0$ for all neighboring particle pairs. $\phi_{\max }^{\text {core }}$ can be identified with random close packing giving a value of 0.63 . When discussing our experimental results, we use the effective volume fraction $\phi_{\text {eff }}$ instead of $\phi_{c}$, where $\phi_{\text {eff }}$ is defined as the volume fraction of the particles including the undeformed polymer layer

$$
\phi_{\mathrm{eff}}=\left(\frac{R_{c}+L}{R_{c}}\right)^{3} \phi_{\text {core }} .
$$

Note that for particle configurations with $H_{\mathrm{av}}<2 L$ the polymer layers are deformed; so, $\phi_{\text {eff }}$ overestimates the volume fraction for those configurations.

The method for calculating $\mu_{\text {lub }}$ has been used to model the high shear viscosity for concentrated suspensions [6]. We added the solvent viscosity and single-particle contribution to $\mu_{\text {lub }}$ to obtain a $\eta_{\infty}\left(\phi_{\text {eff }}\right)$ description, which satisfies the expected behavior at low and high concentrations. The expression for the high shear viscosity $\eta_{r, \infty}$ then becomes

$$
\eta_{r, \infty}=1+\frac{5}{2} \phi_{\mathrm{eff}}+\frac{\mu_{\mathrm{lub}}}{\mu} .
$$

By using $\phi_{\text {eff }}$ in Eq. (18) we have anticipated that at low concentrations the penetration of the solvent into the polymer layer can be ignored.

\section{EXPERIMENTAL SECTION}

\section{A. Materials}

All measurements were performed with a suspension of particles that consist of a silica core coated with a layer of end-grafted PDMS. The solvent was heptane that is a good solvent for PDMS at the temperature where all measurements were performed, $25{ }^{\circ} \mathrm{C}$. Both synthesis and characterization results are elaborately reported by Nommensen $\mathrm{et}$ al. [8]. Here we summarize these results briefly.

The particles were synthesized in two steps. First, bare silica cores were prepared according to the method of Stöber et al. [9]. PDMS molecules with $M_{n}=8 \times 10^{4} \mathrm{~g} / \mathrm{mol}$ and $M_{w} / M_{n}=1.4$ were grafted in a separate step to the bare particles using the method of Auroy et al. [10]. This two-step method allows the characterization of the silica cores before the PDMS is grafted to them.

The radius of the silica cores $\left(R_{c}\right)$ was obtained using both transmission electron microscopy and static light scattering giving, respectively, $80 \mathrm{~nm}$ with a polydispersity of $8 \%$ and $82 \pm 5 \mathrm{~nm}$. The hydrodynamic radius of the grafted particles (in dilute solutions) $R_{h}$ was determined using dynamic light scattering. Its magnitude was $140 \pm 5 \mathrm{~nm}$ indicating a polymer layer thickness $L$ of $58 \pm 7 \mathrm{~nm}$.

The hydrodynamic specific volume (in dilute solutions) $q_{h}$ was determined via intrinsic viscosity measurements using an automated (Schott) Ubbelohde capillary viscometer where we set the intrinsic viscosity to that of a hard sphere. Comparing $q_{h}$ with the specific volume of the bare particles (determined by mass-density measurements), the ratio $R_{h} / R_{c}$ can be calculated. We obtained the value of $1.8 \pm 0.1$ in good agreement with the $1.7 \pm 0.1$ found with light scattering.

We estimated the area per PDMS molecule at the silica surface to be $16 \mathrm{~nm}^{2}$ by combining results for the massfraction polymer/particle, the number averaged polymer molecular weight, the specific volume of the core, and the radius of the core. The area per polymer molecule at the periphery can be obtained from this by using the ratio $R_{h} / R_{c}$, which gives $49 \mathrm{~nm}^{2}$. The lateral distances $(4-7 \mathrm{~nm})$ between polymer chains are much smaller than the layer thickness, which indicates that the molecules are strongly stretched, and hence form a brushlike structure.

All sample suspensions were made from a single stock. Concentrated samples were made by centrifuging a weighed amount of stock, pipetting off the calculated weight of heptane, and subsequently vigorously shaking the suspension. From the particle weight fractions $w$, weight concentrations $c$ were calculated according to $1 / c=\left(v_{p}-v_{s}\right)+v_{s} / w$ with $v_{p}$ and $v_{s}$ the gravimetrical specific volumes of, respectively, the particles and the solvent. The effective volume fraction is calculated from the weight concentration using the relation $\phi_{\text {eff }}=q_{h} c$.

\section{B. Rheological measurements}

Flow curves were measured with a Contraves Low Shear 40 using a Couette geometry with inner and outer radii of, 
respectively, 5.5 and $6.0 \mathrm{~mm}$. The shear rate $\dot{\gamma}$ was varied from $2 \times 10^{-4}$ to $80 \mathrm{~s}^{-1}$. Prior to the measurements, the sample was presheared at a shear rate of $80 \mathrm{~s}^{-1}$ for several minutes. It was checked that steady-state conditions were reached by monitoring the viscosity at constant shear rate versus time. For concentrated suspensions this could take up to 20 minutes at low shear rates.

More flow curves together with linear viscoelastic moduli were measured with a Bohlin VOR rheometer using a coneplate geometry of diameter $60 \mathrm{~mm}$ and angle $1^{\circ}$. The samples were presheared at $81 \mathrm{~s}^{-1}$ with short test measurements in between to monitor the time dependence of the elastic modulus $G^{\prime}$ at $1 \mathrm{~Hz}$. Measurements were started as soon as this quantity became stable. The shear rate was varied from 0.01 to $6 \times 10^{3} \mathrm{~s}^{-1}$. Frequency sweeps were carried out from 0.001 to $10 \mathrm{~Hz}$ after the critical shear amplitude, marking the end of the linear regime, had been determined at $1 \mathrm{~Hz}$.

Both the Contraves and the Bohlin were equipped with a homemade vapor lock to prevent a change in concentration during measurements. Especially concentrated suspensions are sensitive to solvent evaporation. Using the vapor locks, no change in concentration was detected even after more than 12 hours.

Three types of rheological behavior are typically observed in our suspensions. Dilute suspensions show a constant viscosity and are referred to as Newtonian liquids. At moderate and high concentrations the viscosity becomes shear rate dependent. A constant viscosity at low shear rate, (i.e., a low shear plateau $\eta_{0}$ ), is followed by a shear thinning region and a high shear rate plateau $\eta_{\infty}$. For highly concentrated suspensions, the low shear plateau is no longer observable. The shear rate dependence of the viscosity at low shear rates can then be described with a power law, with a power of approximately minus 1 indicating the presence of an apparent yield stress.

Flow curves, which show a low shear rate viscosity plateau, were fitted with the Cross relation

$$
\eta=\eta_{\infty}+\frac{\eta_{0}-\eta_{\infty}}{1+\left(\frac{\dot{\gamma}}{\dot{\gamma}_{\text {crit }}}\right)^{m}}
$$

to obtain the magnitude of $\eta_{0}$ and $\eta_{\infty}$.

At concentrations where the apparent yield behavior was observed at low shear rates, the flow curves were fitted with the following empirical relation to find the magnitude of $\eta_{\infty}$ and the apparent yield stress $\tau_{y}$ :

$$
\eta=\eta_{\infty}+\frac{\tau_{y}}{\dot{\gamma}}+b \dot{\gamma}^{-m}
$$

This relation is the Herschel-Bulkley model to which a high shear viscosity has been added.

For shear rates larger than $\dot{\gamma}_{\text {crit }}$, Eq. (19) can be simplified (since $m>0$ ) to

$$
\eta \approx \eta_{\infty}+\left(\eta_{0}-\eta_{\infty}\right) \dot{\gamma}_{\mathrm{crit}}^{m} \dot{\gamma}^{-m}=\eta_{\infty}+b \dot{\gamma}^{-m}
$$

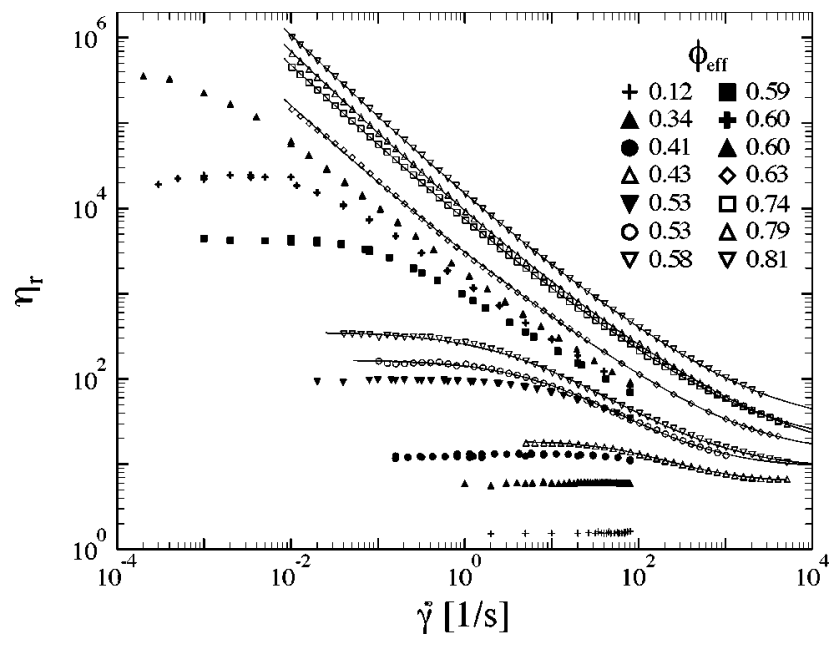

FIG. 2. Flow curves of the silica/PDMS system in the effective volume range $0.12-0.81$ measured with a Contraves (closed symbols) and with a Bohlin (open symbols). The lines represent the fits to Eqs. (19) and (20).

indicating that no discontinuity in the obtained $\eta_{\infty}$ can be expected due to the change over between the fit equations. This was checked by fitting flow curves that show a low shear viscosity with both Eqs. (21) and (20) in the shear rate range $\dot{\gamma} / \dot{\gamma}_{\text {crit }}>10$. The magnitude found for $\eta_{\infty}$ was virtually identical in both fits. The value obtained for $\tau_{y}$, which fitted these curves, deviated not significantly (within the error range) from zero. From this we conclude that the high shear limiting behavior is equally well described by both equations.

The accuracy of the fitted high shear plateaus depends strongly on the upper limit of the measured range in shear rate. The highest accessible shear rate on the Bohlin was sufficient for a satisfactory accuracy but the upper limit in shear rate range of the Contraves is too small. Hence, only results of the Bohlin are considered in the discussion about the high shear viscosity.

Results for the zero frequency elastic modulus $G_{0}^{\prime}$ and for the high frequency elastic modulus $G_{\infty}^{\prime}$ were obtained by taking the value for $G^{\prime}$ at the lowest and highest measured frequency $(0.001 \mathrm{~Hz}, 20 \mathrm{~Hz})$. This method underestimates $G_{\infty}^{\prime}$ since the measured frequency sweeps showed a small slope even at the highest concentration.

\section{RESULTS AND DISCUSSION}

\section{A. Flow curves}

The relative steady shear viscosity $\eta_{r}$ is shown in Fig. 2 as a function of shear rate $\dot{\gamma}$ for effective volume fractions in the range of 0.12 to 0.81 . A drastic change over in rheological behavior occurs around $\phi_{\text {eff }}=0.60$. Below 0.60 a low shear viscosity plateau is observed, followed by a shear thinning region and the onset of a high shear viscosity plateau. These flow curves could be fitted well with Eq. (19). Above $\phi_{\text {eff }}=0.60$ the flow curves indicate the presence of an apparent yield stress. An onset of a high shear viscosity is also observed. Equation (20) fits these flow curves with excellent accuracy. 


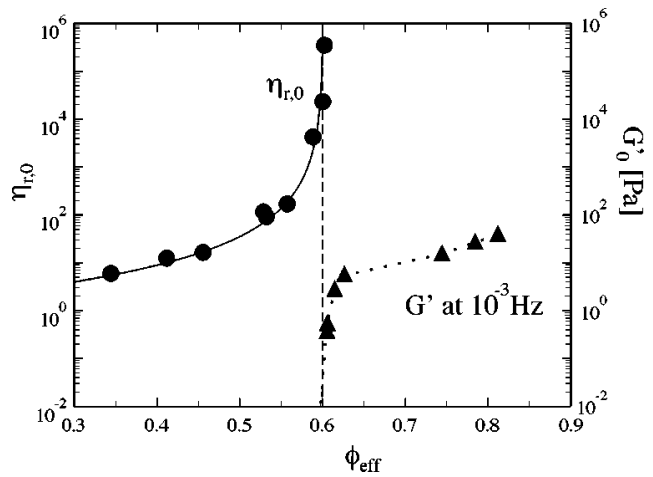

FIG. 3. When the relative low shear viscosity (circles) and the elastic modulus at the lowest measurable frequency $10^{-3} \mathrm{~Hz}$ (triangles) are plotted versus the effective volume fraction, the transition from liquid to solidlike behavior is clearly seen. The solid line represents the fit to Eq. (22).

This qualitative behavior of the flow curves appears to be typical for suspensions consisting of colloidal particles that interact repulsively due to their steric stabilization layers $[2,3,11]$. The effective volume fraction $\phi_{\text {eff }}=\phi_{m}$ where the transition occurs, differs from system to system. Although all $\phi_{\text {eff }}$ 's are defined in the same way, reported values for $\phi_{m}$ range from about 0.5 up to 0.71 .

The effective volume fraction as defined in Sec. III is based on experiments with dilute suspensions. The use of this definition at high concentrations may result in effective volume fractions higher than corresponding to the maximum packing for hard spheres, in the case the particles can be deformed. Indeed we were able to measure samples with $\phi_{\text {eff }}$ up to 0.81 showing the deformability or softness of the particles.

\section{B. Rheological behavior at low shear rates}

The low shear viscosity is plotted versus the effective volume fraction in Fig. 3 together with the elastic modulus $G_{0}^{\prime}$. The low shear viscosity diverges at $\phi_{\text {eff }}=0.60$ and at that volume fraction the elastic modulus becomes nonzero. This demonstrates clearly the transition from liquid to solidlike behavior.

The low shear viscosity, observed for $\phi_{\text {eff }}$ up to 0.60 , can be fitted well with Quemada's expression [12],

$$
\eta_{r}=\left(1-\frac{\phi_{\text {eff }}}{\phi_{m}}\right)^{-2}
$$

with $\phi_{m}=0.60 \pm 0.02$. Despite the softness of our particles this result is within the range of values obtained for hardsphere systems fitted with Eq. (22) [13]. In a previous study [8], the linear viscoelastic behavior was also found to be in agreement with that of hard spheres, up to $\phi_{\mathrm{eff}} \approx \phi_{m}$.

These findings indicate that our hairy sphere suspensions behave like a hard-sphere suspension until the average distance between the particle centers becomes close to twice the outer radius of the particles.

In previous studies on monodisperse soft sphere suspensions by D'Haene [2] and Jones et al. [3], it was found that $\phi_{m}$ shifts to higher values when the ratio $R_{c} / L$ decreases. This ratio is 1.4 for our silica/PDMS particles, which is
TABLE I. Experimental results for the apparent yield stress and elastic modulus with their corresponding effective volume fraction.

\begin{tabular}{ccc}
\hline \hline$\phi_{\text {eff }}$ & $\tau_{y}(\mathrm{~Pa})$ & $G_{\infty}^{\prime}(\mathrm{Pa})$ \\
\hline 0.61 & 0.20 & 16 \\
0.63 & 0.47 & 23 \\
0.74 & 1.6 & 53 \\
0.78 & 2.5 & 66 \\
0.81 & 3.8 & $1.0 \times 10^{2}$ \\
\hline \hline
\end{tabular}

much smaller than any of the particles used in the studies mentioned above (smallest ratio is 4.7 [2]). Our result for $\phi_{m}$, however, does not fit in these observations since it is much smaller than the magnitude for $\phi_{m}$, found for the system with ratio 4.7.

At volume fractions larger than $\phi_{m}$ our flow curves indicate the existence of an apparent yield stress (i.e., a stress plateau at low shear rates). The apparent yield stress as obtained from fitting to Eq. (20) strongly depends on the effective volume fraction: see Table I. It could be fitted well with the power law function

$$
\tau_{y}=b \phi_{\mathrm{eff}}^{m}
$$

with an exponent $m$ of $10 \pm 1$. This magnitude of $m$ is still smaller then any of the exponents found by D'Haene for his range of PMMA/PHS systems $\left(R_{c} / L\right.$ from 4.7 to 175$)$.

When $\tau_{y}$ is plotted versus $G_{\infty}^{\prime}$ (Table I also contains $G_{\infty}^{\prime}$ data), a linear dependence is obtained with a slope 0.041 \pm 0.006 . This linear dependence is also seen in other sterically stabilized suspensions. D'Haene observed a slope $0.03 \pm 0.01$ for his suspensions. This slope was estimated theoretically by Buscall [14] to be 0.02 for suspensions of soft repulsive spheres where both $\tau_{y}$ and $G_{\infty}^{\prime}$ are dominated by the interparticle potential. Considering the crudity of the theoretical approach and the error in extrapolating $G^{\prime}(\omega)$ to infinite $\omega$, the agreement of the various results within the same order of magnitude leads to the conclusion that the results corroborate each other.

\section{Shear thinning}

In suspensions with $\phi_{\text {eff }}$ larger than 0.4, shear thinning has been observed. With increasing volume fractions, the shear thinning is more pronounced and starts at lower shear rates. This last phenomenon can be monitored using the critical shear rate $\dot{\gamma}_{\text {crit }}$, which is the shear rate where the viscosity equals $\frac{1}{2}\left(\eta_{0}+\eta_{\infty}\right)$.

Anticipating a correlation with the long time diffusion and the low shear viscosity, D'Haene scaled $\dot{\gamma}_{\text {crit }}$ using

$$
\dot{\gamma}_{r}=\frac{\eta_{0} \dot{\gamma}_{\text {crit }}\left(R_{c}+L\right)^{3}}{k T} \text {. }
$$

Plotting $\dot{\gamma}_{r}$ as a function of the volume fraction, the data of several of their systems with different $R_{c}$ collapsed to a region between 0.1 and 1 . When plotting our $\gamma_{\text {crit }}$, obtained from the fit to Eq. (19); in this fashion the scaled data fall in the same region. 


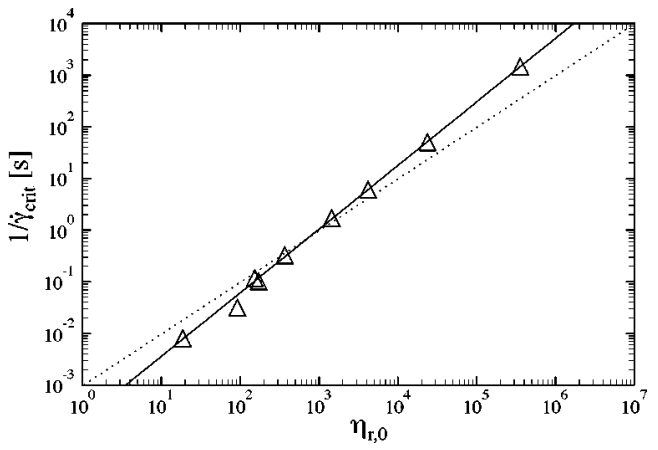

FIG. 4. Reciprocal critical shear rate versus the relative low shear viscosity. The solid line is a power law fit, the dotted line represents the best linear fit.

Scaling with Eq. (24) indicates a linear relation between $1 / \dot{\gamma}_{\text {crit }}$ and $\eta_{0}$. For our silica/PDMS particles we found a slightly nonlinear but strong correlation, as can be seen in the plot of $1 / \dot{\gamma}_{\text {crit }}$ vs $\eta_{0}$ in Fig. 4 . An excellent fit was obtained with a power law function with power $\alpha=1.23 \pm 0.04$. Fitting to a linear function (also plotted in Fig. 4) results in poor fit.

\section{Rheological behavior at high shear rate}

In this range of shear rate we obtained experimental data for the viscosity, which will be compared to the model calculations of Sec. II. In the first subsection we give the algorithm used in the viscosity calculations after which we test the model by comparing to results for hard-sphere suspensions. In the second subsection the experimental data is given together with the comparison to the viscosity calculations.

\section{Viscosity calculations}

The viscosity $\left(\eta_{\infty}\right)$ calculations were performed by first solving Eq. (7) and integrating Eq. (13) analytically to obtain the flux $\bar{Q}$ as a function of $\bar{h}$ and $\bar{h}_{L}$. Equations (8) and (9) were used to make $\bar{Q}$ a function of $\bar{r}$ after which the lubrication force was calculated by integrating Eq. (14) numerically. Finally the viscosity was obtained using Eqs. (15)(18).

Our algorithm to calculate the viscosity makes it possible to investigate the influence of adding higher-order terms in the description of the gap geometry in Eqs. (8b) and (9b). The introduction of the next term increases the local gap thickness for $r>0$ causing a lowering of the pressure gradient needed for the squeezing flow. Hence, the calculated lubrication force decreases and, therefore, also the viscosity.

As a test case we calculated the viscosity for hard spheres using both gap geometries and compared it to experimental high shear data for hard-sphere particles compiled from literature $[13,15]$. Expanding only up to the quadratic term in Eq. (8b), the calculated viscosities are much larger than the reference data, especially at moderate concentrations: see Fig. 5. By incorporating the next term, Eq. (8b) becomes

$$
h(r) \approx H+\frac{r^{2}}{R_{c}}+\frac{r^{4}}{4 R_{c}^{3}}+O\left(r^{6}\right) .
$$

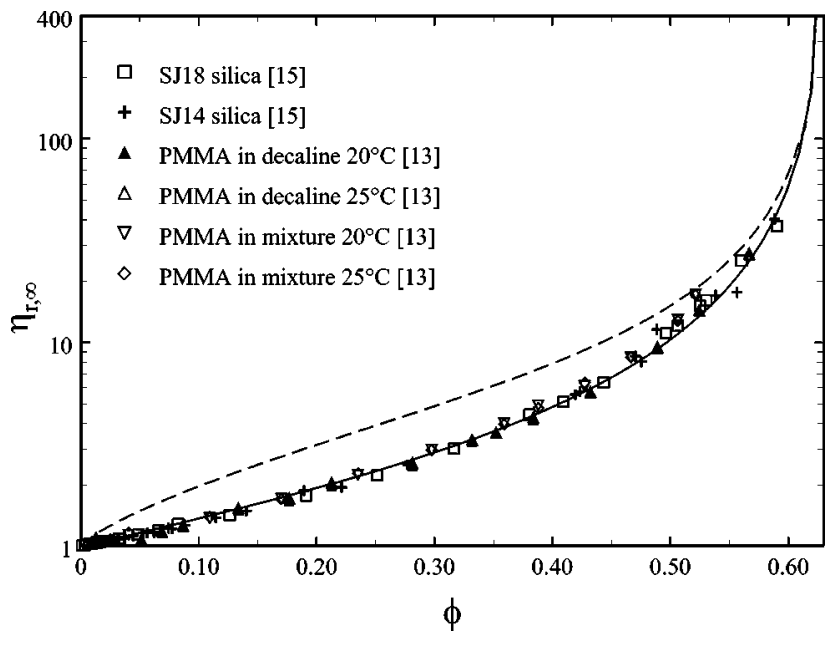

FIG. 5. Experimental relative high shear viscosity for hardsphere suspensions compiled from literature $[13,15]$. The lines are calculations with a gap geometry description up to quadratic term (dashed) and using higher-order terms (solid).

Using this, instead of Eq. (8b), the results of the calculations describe the hard-sphere reference data excellently if the value for $\phi_{\max }^{\text {core }}$ is set to 0.63 . Obviously, using this description is also attractive for hairy spheres. To be consistent in our approach, Eq. (9b) also has to be replaced,

$$
h_{L}(r) \approx H-2 L+\frac{r^{2}}{R_{c}+L}+\frac{r^{4}}{4\left(R_{c}+L\right)^{3}}+O\left(r^{6}\right) .
$$

We used Eqs. (25) and (26) in all further calculations.

The calculations for the hairy spheres can now be tested by comparing them to known cases. When the permeability $\delta^{2}$ is very large, the disturbance of the solvent flow due to the polymer layer can be neglected so the behavior of the hairy particles should approach the behavior of hard spheres with radius $R_{c}$. In the other limit, very small $\delta^{2}$, the solvent flow can penetrate the polymer layer only up to negligible depth so the hairy spheres should show behavior like that of hard spheres with radius $R_{c}+L$. When Eqs. (25) and (26) are used for the gap geometry, the calculations indeed show the expected behavior. This is illustrated in Fig. 6 where the lubrication forces of hairy sphere systems with very large and very small $\delta$ and of the appropriate hard-sphere systems are plotted versus the gap thickness. A hairy particle system

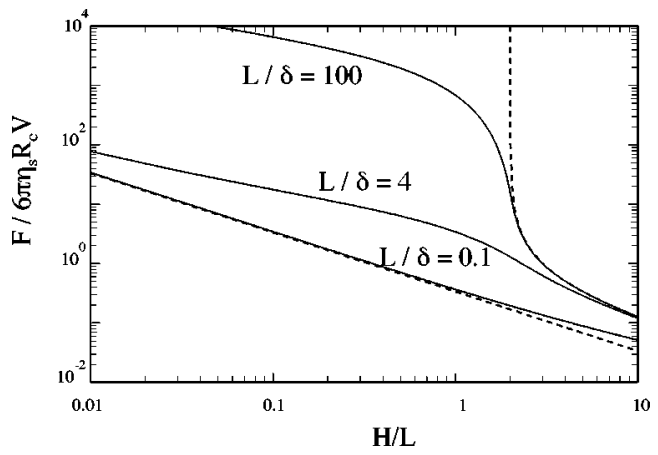

FIG. 6. The lubrication force vs gap thickness for our hairy particles $\left(R_{c} / L=1.4\right)$ for several $\delta$ (solid lines) together with results for hard spheres with radii $R_{c}$ and $R_{c}+L$ (dashed lines). 


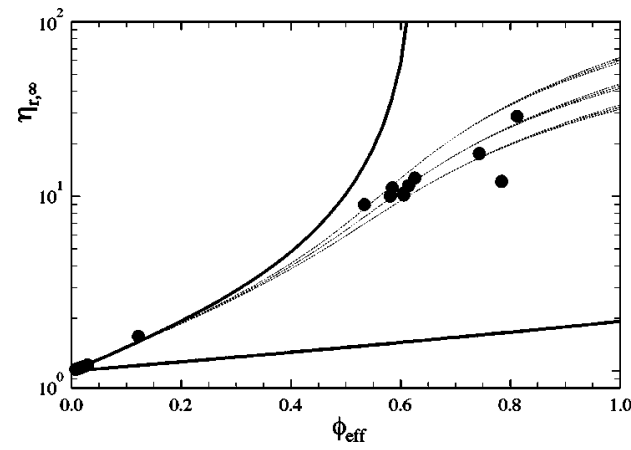

FIG. 7. Experimental relative high shear viscosity (circles) compared to calculations (dashed lines) for $\delta=4,5$, and $6 \mathrm{~nm}$ (respectively, the top, middle, and bottom set of curves) and several solvent qualities: $n=\frac{1}{2}, \frac{3}{4}, 1$. Solid lines represent hard-sphere bounds for our hairy particles. Effects of the solvent quality appear only at very large $\phi_{\text {eff }}$.

with moderate $\delta$ is also plotted. For large gap thicknesses, the lubrication force of this last system has a magnitude that is close to the hard-sphere system with radius $R_{c}+L$. But for small gap thickness, it is close to the hard-sphere system with $R_{c}$.

The influence of the solvent quality in the calculations was also tested. In our model the solvent quality is only incorporated as parameter $n$ in the description of the compressed polymer brush. Three values of $n$ have been given in Fig. 7. For slightly compressed brushes, almost no influence is seen. Only when the brushes are strongly compressed, the solvent quality becomes an important parameter. In the range of experimentally accessible $\phi_{\text {eff }}$ 's, the brushes are only slightly compressed so any influence of the solvent quality can be ignored.

\section{Comparison of experimental data to the viscosity calculations}

Many of our flow curves show the onset of a high shear viscosity plateau. The magnitude of $\eta_{\infty}$ was obtained by extrapolating the flow curves using Eqs. (19) or (20). In Fig. 7, $\eta_{\infty}$ is plotted versus $\phi_{\text {eff }}$. The calculations for hard-sphere systems with radii $R_{c}$ and $R_{c}+L$ are also plotted in this figure. At low concentrations, the silica/PDMS system behaves like a hard-sphere system with radius $R_{c}+L$. In Fig. 7 the experimental data are also compared to calculations using Eq. (18) with $R_{c} / L=1.4$ and $\delta=4,5$, and $6 \mathrm{~nm}$. The calculation with $\delta=5 \mathrm{~nm}$ describes the experimental data fairly well at both low and high volume fraction.

The magnitude of $\delta$ can be identified with the characteristic "mesh size" of the polymers [6] that can be related to average distance between grafting sites (or equivalently the average area per molecule) in the case that the polymers are stretched [16]. Based on our particle characterization, we expect a $\delta$ in the range from 4 to $7 \mathrm{~nm}$, which agrees well with the $5 \mathrm{~nm}$ obtained from the fit.

With the improved description for the gap geometry, it is interesting to reanalyze the high shear viscosities obtained for other hairy sphere dispersions $[2,4]$.

We calculated the $\eta_{\infty} / \phi_{\text {eff }}$ curve for various monodisperse PMMA/PHS systems of D'Haene. The systems with $R_{c} / L$ of 4.7 and 7.2 could be fitted fairly well. It turned out that for the particles with larger $R_{c} / L, 21$ and 26, the experi-

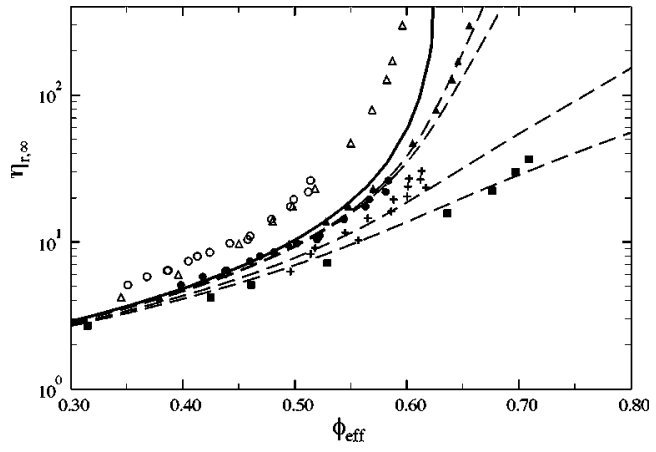

FIG. 8. Experimental data of the D'Haene for $\eta_{\infty}$ compared to our model calculations with $L / \delta=5.5$ (dashed lines). $R_{c} / L=4.7$ (squares), 7.2 (crosses), 21 (circles), and 26 (triangles). The open symbols represent the original data before volume fraction rescaling. Solid lines represent the hard-sphere bounds.

mental data were not within our hard-sphere bounds (corresponding to particles with a fully permeable respectively totally impermeable polymer layer). Assuming that the errors in the viscosity measurements were small and that the PMMA/PHS particles can be described as purely repulsive spheres, this would suggest a rescaling of the volume fractions.

A suitable criterion for such a rescaling can be obtained from the fact that for low concentrations the viscosity does not depend on the penetration depth (as was observed in our calculations). Therefore, we multiplied all volume fractions with a scale factor that causes the calculated $\eta\left(\phi_{\text {eff }}\right)$ curves to coincide with the experimental curve in the concentration range where softness effects do not show up yet. After separately rescaling the data sets for $R_{c} / L=21$ and 26 in such a manner, the calculations describe the experimental data well.

Phan et al. [13] also rescaled the volume fraction of experimental data of D'Haene's hard-sphere systems $\left(R_{c} / L\right.$ $=26$ and 68). The criterion they used for scaling was that the relative high shear viscosity at a volume fraction of 0.5 should be equal to 11.5. After rescaling, the volume fraction dependence of $\eta_{\infty}$ is similar to data of other hard-sphere systems. The scale factor they used is slightly smaller then our scale factor that can be expected since we modeled the PMMA/PHS particles as soft spheres instead of hard spheres.

In Fig. 8 the results of the calculations are plotted together with the experimental data. The hard-sphere bounding curves are also plotted. The inset shows the original data. All the calculated curves that describe the data well have a magnitude of the penetration depth in the order of $1 \mathrm{~nm}$, which is roughly $10 \%$ of the layer thickness.

Calculations were also performed for the PS/PVA systems of Neuhäusler and Richtering with $R_{c} / L$ of 1.52 and 1.75. The results of these calculations give a poor description. They are plotted in Fig. 9 where we transformed the core volume fraction to an effective volume fraction using Eq. (17). In their article, Neuhäusler and Richtering reported that there might be a concentration dependence of the layer thickness. If we use a larger but constant ratio $R_{c} / L$, the calculations describe the experimental data surprisingly well. A possible explanation is that the number of polymers per particle is smaller at high volume fractions than at low volume fractions, where the layer thicknesses were determined. 


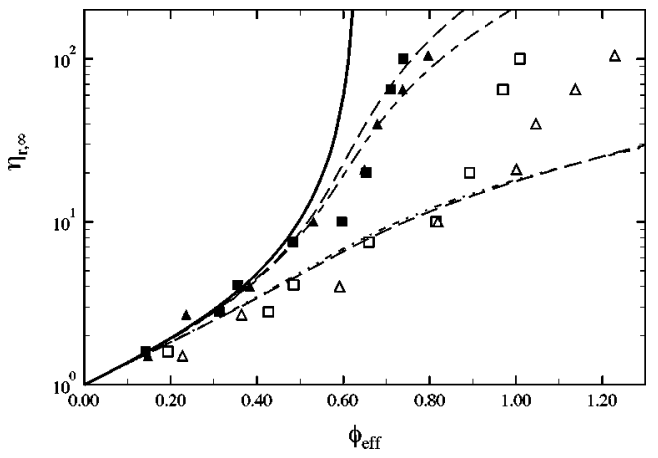

FIG. 9. Experimental data of Neuhäusler and Richtering together with our calculations for $R c / L 1.52$ (triangles) and 1.75 (squares). The solid symbols represent the data after rescaling $R_{c} / L: 1.52 \rightarrow 2.3$ and $1.75 \rightarrow 2.4$. See text for details.

\section{CONCLUSIONS}

The rheological behavior of a suspension of monodisperse spheres coated with a polymer brush was investigated experimentally with an emphasis on the steady shear rate viscosity measurements. Both the particle core and the polymer layer could be characterized well since the polymers were grafted to the particles after the synthesis of the cores. The chemical bonding of the polymer molecules to the core surface prevents changes in polymer layer properties in time. These together make this suspension to a good model system for studying the influence of the polymer brush on the rheology.

The rheological data show a liquid to solidlike transition at an effective volume fraction $\phi_{m}=0.60$. Below $\phi_{m}$, low shear viscosity plateaus were observed. These plateaus were no longer observable above $\phi_{m}$; instead, an apparent yield stress was seen. The concentration dependence of the low shear viscosity $\eta_{0}$ indicates that the silica/PDMS particles can be modeled as hard spheres up to $\phi_{\text {eff }}$ where the magnitude of $\eta_{0}$ diverges.

The concentration dependence of the high shear viscosity $\eta_{\infty}$ could be described well with a hydrodynamic model consisting of a summation over the solvent viscosity, the single particle contribution, and the viscosity due to lubrication. This last contribution is based on the lubrication model of Potanin and Russel to which we added some modifications. First, the lubrication contribution for hard spheres was calculated with an improved description for the gap geometry, resulting in a excellent description of compiled literature data for hard spheres. Second, the differences in curvature between the core surface and the polymer layer were taken into account. Comparing our experimental data to the calculations, we used only one fit parameter, the penetration depth $\delta$. The best fit was found for $\delta=5 \mathrm{~nm}$, which is in the range of theoretical estimates for $\delta$. Using this model, experimental data for other hairy sphere suspensions could also be described quantitatively after small adjustments in the particle model parameters for some of these systems. The description of some of these systems improved after small adjustments of the experimental data.

\section{ACKNOWLEDGMENTS}

This work is part of the research program of the Foundation for Chemical Research (SON) with financial support from the Netherlands Organization for Scientific Research (NWO). We thank J.S. Lopulissa for synthesizing the silica/ PDMS particles, J. Mewis, S.-E. Phan, and W. Richtering for providing us with their data, and W.B. Russel for valuable discussions.
[1] J. Mellema, Curr. Opin. Colloid Interface Sci. 2, 411 (1997).

[2] P. D'Haene, Ph.D. thesis, Katholieke Universiteit Leuven, Belgium, 1992 (unpublished).

[3] D. A. R. Jones, B. Leary, and D. V. Boger, J. Colloid Interface Sci. 150, 84 (1992).

[4] S. Neuhäusler and W. Richtering, Colloids Surf., A 97, 39 (1995).

[5] G. H. Fredickson and P. A. Pincus, Langmuir 7, 786 (1991).

[6] A. A. Potanin and W. B. Russel, Phys. Rev. E 52, 730 (1995).

[7] N. A. Frankel and A. Acrivos, Chem. Eng. Sci. 22, 847 (1967).

[8] P. A. Nommensen, M. H. G. Duits, J. S. Lopulissa, D. van den Ende, and J. Mellema, Prog. Colloid Polym. Sci. 110, 144 (1998).
[9] W. Stöber, A. Fink, and E. Bohn, J. Colloid Interface Sci. 26, 62 (1968).

[10] P. Auroy, L. Auvray, and L. Leger, J. Colloid Interface Sci. 150, 187 (1992).

[11] D. A. R. Jones, B. Leary, and D. V. Boger, J. Colloid Interface Sci. 147, 479 (1991).

[12] D. Quemada, Rheol. Acta 16, 82 (1977).

[13] S.-E. Phan, W. B. Russel, Z. Cheng, J. Zu, P. M. Chaikin, J. H. Dunsmuir, and R. H. Ottewill, Phys. Rev. E 54, 6633 (1996).

[14] R. Buscall, J. Chem. Soc., Faraday Trans. 87, 1365 (1991).

[15] J. C. v. d. Werff and C. G. de Kruif, J. Rheol. 33, 421 (1989).

[16] S. Alexander, J. Phys. (France) 38, 977 (1977). 